\title{
Beobachtungen von Cometen und Planeten auf der Leipziger Sternwarte.
}

Von Herrn Prof. Bruhns, Director der Sternwarte.

Bis zur Vollendung des für die hiesige Sternwarte bestellten grossen Aequatoreals mit 12-füssigem Fernrohr befand. sich in der Kuppel der 6-füssige Refractor von Utzschneider $\mathcal{S}$ Fraunhofer mit einer Objectivöffnung von 52 Linien. Mit einem von den Herren Pistor $\$$ Martins dazu gefertigten Fadenmikrometer und fast immer angewandter 140 facher Verg'össerung babue ich die folgenden Beobachtungen ausgeführt.

Resultate aws: dien: Beobachtungen.

Comet 11.1861 .

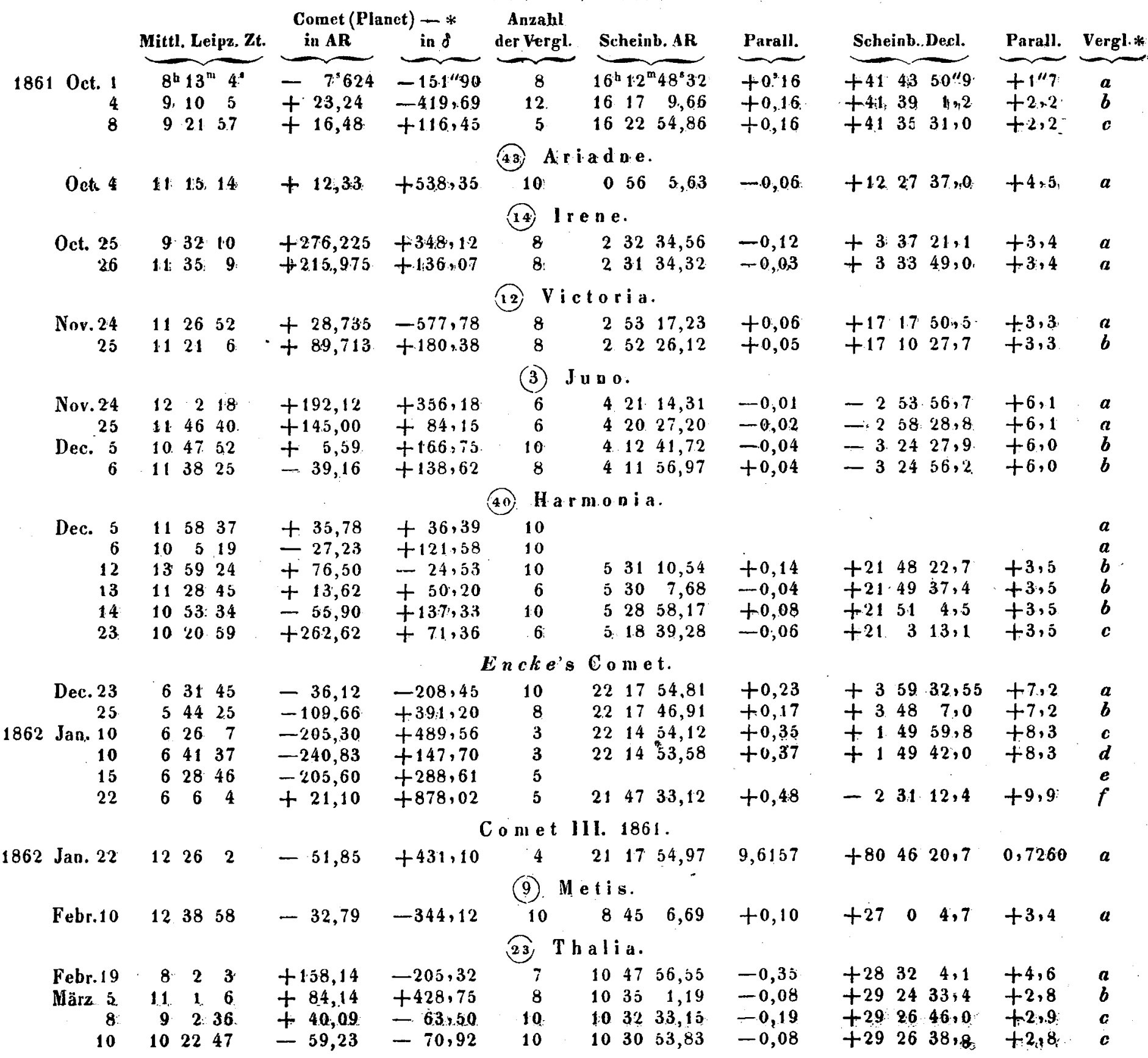




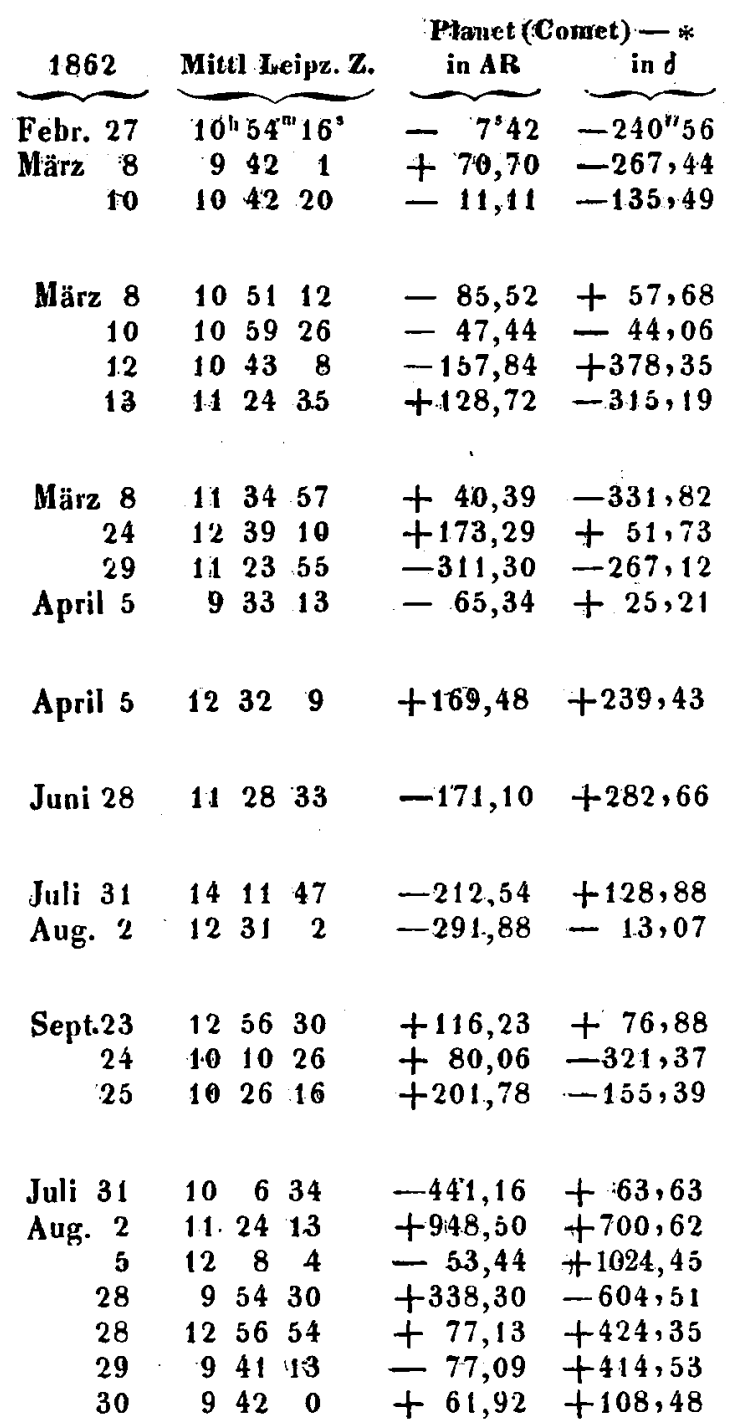

\begin{tabular}{|c|c|c|}
\hline $\begin{array}{l}\text { Amaihi } \\
\text { d. Vergl. }\end{array}$ & Sclieinb. AdR & Parallaxe \\
\hline$A$ & $10^{\mathrm{h}} 10^{\mathrm{m}} 58^{\mathrm{s}} 25$ & $-0^{5} 03$ \\
\hline$g$ & $10 \quad 420,79$ & $-0 ; 05$ \\
\hline 11 & 10256,51 & $-0,01$ \\
\hline
\end{tabular}

(29) Amphitrite.

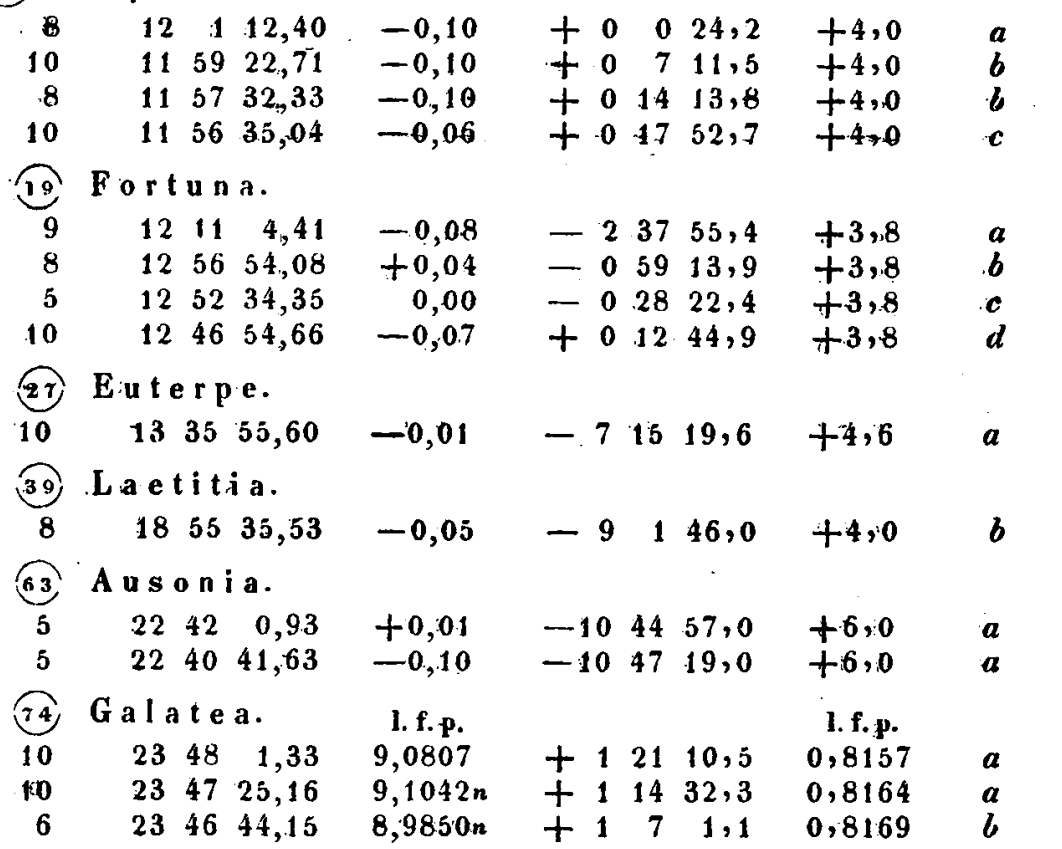

Comet II. 1862 .

8
5
8
4
3
8
8

$$
\begin{array}{rrr}
5 & 53 & 57,65 \\
6 & 3 & 22,93 \\
6 & 23 & 4,33 \\
15 & 31 & 38,30 \\
15 & 32 & 22,52 \\
15 & 36 & 42,51 \\
15 & 41 & 10,00
\end{array}
$$

$9,3216 n$

$9,8356 n$

$9,9866 n$

9,6560

$9,6175 n$

9,6125

9,5837

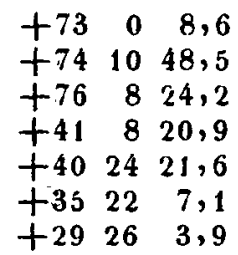

0,8452

0,7952

0,7447

0,5972

0,8286

0,6348

0,6864

Comet III. 1862 .

$$
\begin{array}{rr}
-38,63 & +766,40 \\
-\quad 5,10 & +186,69 \\
-\quad 1,39 & +473,12 \\
-238,07 & +212,74
\end{array}
$$

$$
\begin{array}{rrrr}
9,3932 n & -14 & 5 & 52,3 \\
9,4327 n & -14 & 46 & 15,5 \\
9,4063 n & -16 & 13 & 15,6 \\
9,3378 n & -28 & 34 & 46,6
\end{array}
$$

Parall.

$$
-0,09
$$

$-0,06$

$+0,03$

$-0,31$

$-0,28$

$-0,33$
23821,4
$-21042,5$
$-14025,7$
$+72244,6$
$+72432,9$
$+83140,5$

$\begin{array}{ll}0,8710 & a \\ 0,8676 & b \\ 0,8753 & c \\ 0,9071 & d\end{array}$

Parall.

$\begin{array}{ll}+6,5 & a \\ +6,7 & b \\ +6,9 & c \\ +9,8 & d \\ +9,8 & d \\ +10,2 & e\end{array}$

In der letzten Hälfte des December wurde das grosse Aequatoreal aufgestellt. Dasselbe rubt auf einer gusseisernen Säule, hat 2 Stundenkreise, einen zum Einstellen, einen zur mikroskopischen Ablesung, und ebenso 2 Declinationskreise.
Die Contrebalancirung ist derjenigen am Gothaer Aequatoreal ahnlich. Das Fernrohr hat 12 Fuss Brennweite und 8 Zoll Oeffnung und giebt sehr gute Bilder. Ein Fadenmikrometer mit Feld- und Fadenbeleuchtung lässt sowobl helle als auch 
achwache Gestirne beobachten. Das Fadenkreuz besteht aus 15 parallelen und 5 darauf senkrechten Fäden und ausserdem aus 2 auf einander senkrechten beweglichen Fäden mit Scalen und Mikrometerschrauben. - Da während des Winters die Beobachtungsnächte so rar waren, habe ich das lostrument vorläufig noch nicht so untersuchen können, um sicher zu sein, gute absolute Bestimmungen zu machen. Die folgenden
Beobacbtungen der Cometen und einiger Planeten sind Differentialbeobachtungen mit dem Fadenmikrometer, bei 144 facher Vergrösserung angestellt. Von den 5 Fäden sind entweder. 1, 2 oder 3 zu den Durchgängen benutat, und auf diese Fäden kommt dann eine Declinationseinstellung. Die Anzahl der Vergleichungen in AR und in sind neben einander in der Columne „Anzahl der Vorgl.“ gegeben.

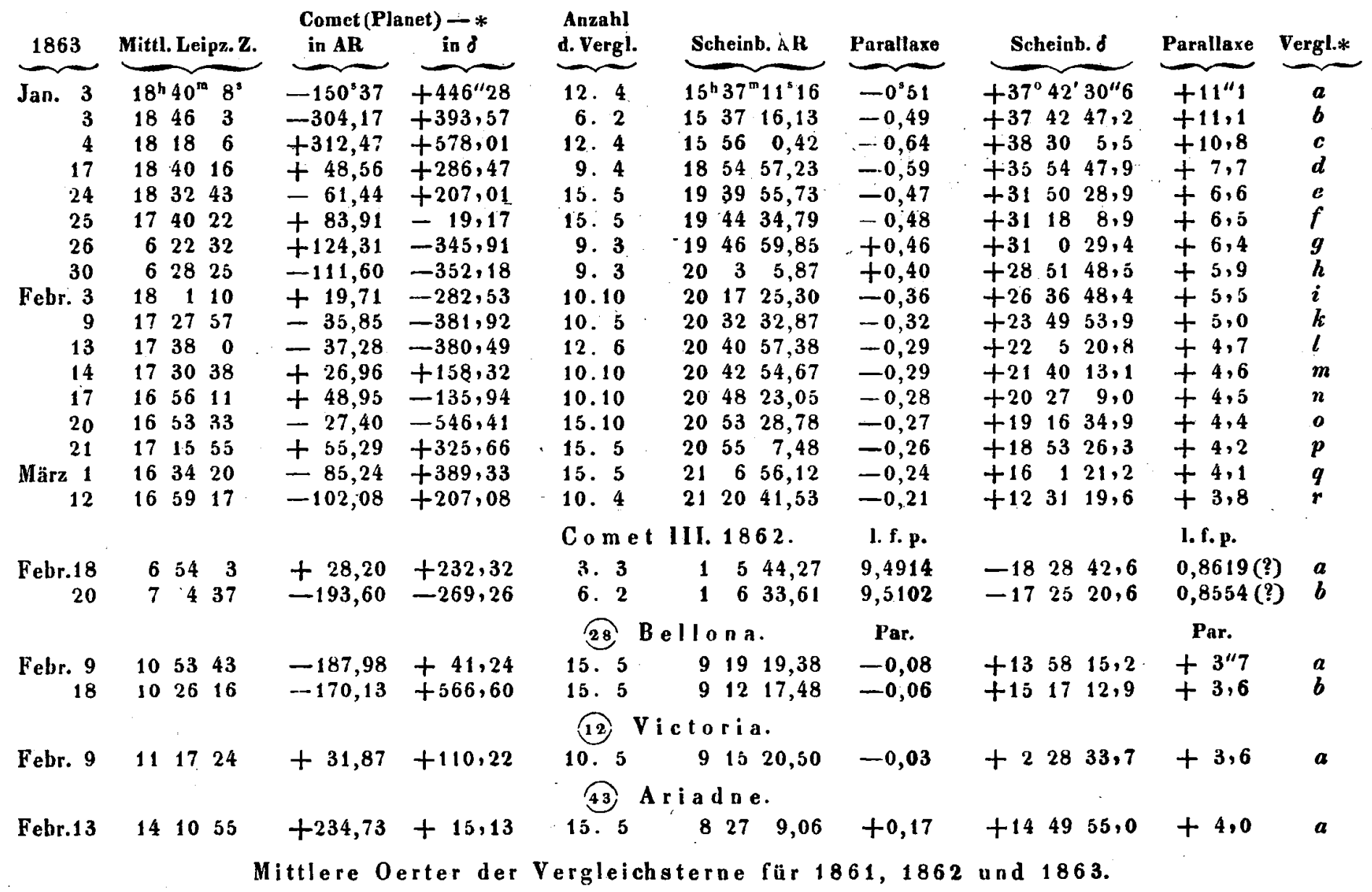

Mittlere Oerter der Vergleichsterne für 1861. Comet 11. 1861.

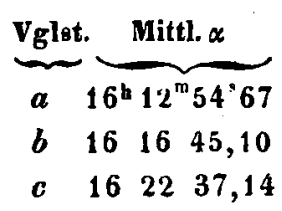

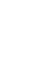

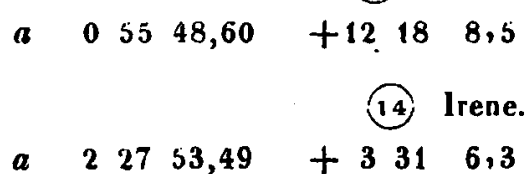

Comet I. 1863 .

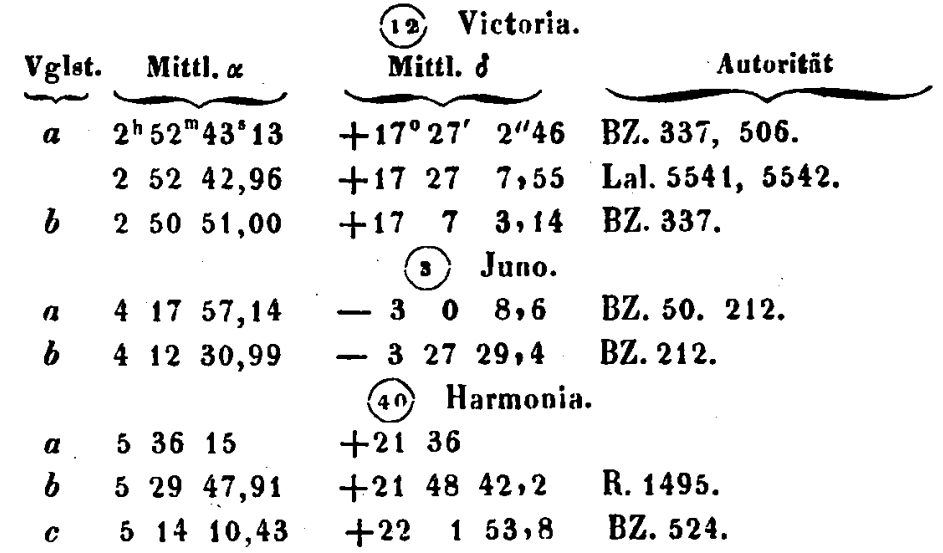



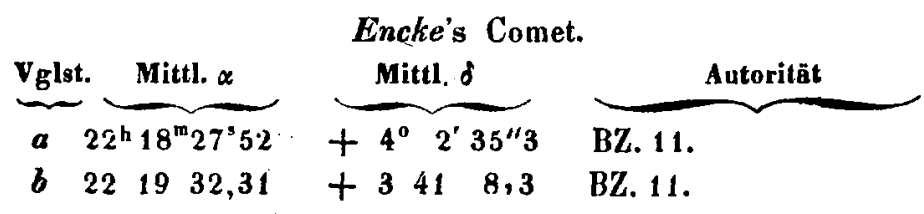

BZ. 11.

BZ. 11.

Mittlere Oerter der Vergleichsterne für 1862.

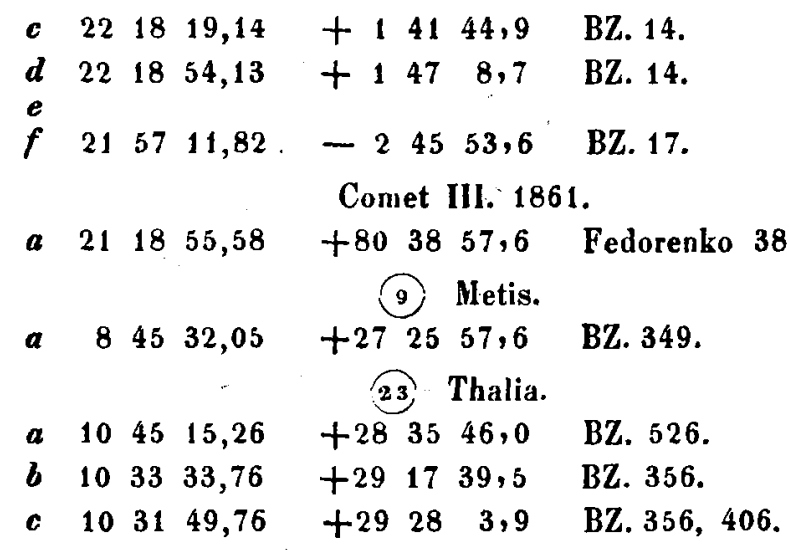

(16) Psyche.

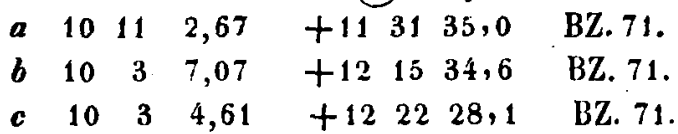

(29) Amphitrite.

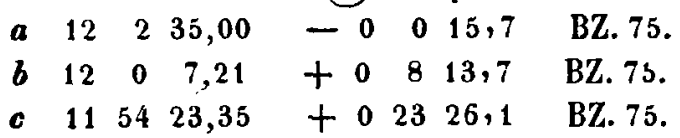

(19) Fortuna.

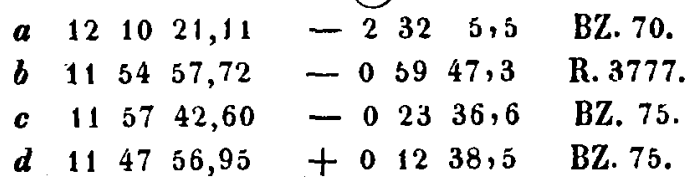

(27) Euterpe.

a $1333 \quad 3,08 \quad-719 \quad 2,7 \quad$ Förster's Best. am Me-

(39) Laetitia. ridiankreise (A. N. 1383).

$\begin{array}{llllllll}a & 18 & 38 & 22,48 & -9 & 6 & 35,6 & \text { BZ. } 256 \text {. }\end{array}$

(63) Ausouia.

$\begin{array}{lllllll}a & 22 & 45 & 29,37 & -104732,5 & \text { BZ. } 186 .\end{array}$

(54) Galateà.

a $23460,55+11926,8$.Lal. 46788,46789 .

b $234317,80+196,0 \quad$ BZ. 34, 36 .

Comet II. 1862.

$a\left\{\begin{array}{llllll}6 & 1 & 15,43 & +7259 & 15,8 & \text { AZ. } 89 . \\ 6 & 1 & 15,43 & +72 & 49 & 15,7\end{array}\right.$ Radcliffe Cat. 1645.

b $54730,64+735918,7 \quad$ AZ. 89 .

c $62355,30+755132,1$ AZ. 170 .

d $152558,26+411819,4$ Mädler 1965 .

Comet II. 1862.

Vglat. Mittl. $\propto$ Mittl. \&

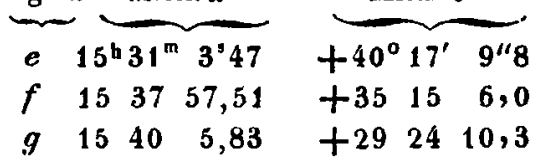

$\begin{array}{llll}a & 13 & 56 & 59,09\end{array}$

$\begin{array}{lllll}b & 13 & 56 & 38,47\end{array}$

c $\quad 13 \quad 57 \quad 4,57$

$\begin{array}{llll}d & 14 & 7 & 2,47\end{array}$

$a\left\{\begin{array}{lll}10 & 36 & 45,52 \\ 10 & 36 & 45,62\end{array}\right.$

$b\left\{\begin{array}{lll}10 & 36 & 52,55 \\ 10 & 36 & 53,23\end{array}\right.$

c $10 \quad 44 \quad 41,49$

$\begin{array}{llll}d & 11 & 38 & 46,04\end{array}$

e $11 \quad 38 \quad 51,43$

$-16 \quad 2053,5$

$-22755,4$

$-21917,6$

$-21921,1$

- 1446,9

71812,9
$+8325,2$

$+83225,2$
Comet III. 1862.

$-141825,3 \quad$ BZ. 242.

$-14498,8 \quad$ BZ. 242.

A.-0̈. $13355,13356$.

$-2838 \quad 6,3 \quad$ Arg.-Oeltzen 13488.

Comet I. 1863 .

- $22753,6 \quad$ Lal. 20706 .

BZ. 68 .

Lal. 20717.

BZ. 68 .

BZ. 68.

B. A. Z. 3982.

BZ. 236.

Mittlere Oerter der Vergleichstene für 1863. Conet I. 1863. $\begin{array}{llll}a & 15 & 39 & 41,86\end{array}$

b $\left\{\begin{array}{lll}15 & 42 & 20,74 \\ 15 & 42 & 20,66\end{array}\right.$

c $15 \quad 50 \quad 48,45$

$\begin{array}{llll}d & 18 & 54 & 9,35\end{array}$

e $19 \quad 4057,65$

$f \quad 194311,11$

$\begin{array}{lllll}g & 19 & 44 & 55,98\end{array}$

$\begin{array}{llll}h & 20 & 4 & 57,75\end{array}$

i $\begin{array}{llll}20 & 17 & 5,67\end{array}$

$\begin{array}{llll}k & 20 & 33 & 8,38\end{array}$

$\begin{array}{llll}l & 20 & 41 & 34,63\end{array}$

$\begin{array}{llll}m \quad 20 & 42 & 27,70\end{array}$

$\begin{array}{llll}n \quad 20 & 47 & 34,03\end{array}$

- $\left\{\begin{array}{lll}20 & 53 & 56,48 \\ 20 & 53 & 55,62\end{array}\right.$

p $\quad 20 \quad 54 \quad 12,01$

$q\left\{\begin{array}{lll}21 & 8 & 21,11\end{array}\right.$

$q\left\{\begin{array}{lll}21 & 8 & 21,11 \\ 21 & 8 & 21,02\end{array}\right.$

$r \quad 212223,0$
$+373518,1$

$+373629,2$

$+373622,9$

$+382039,9$

$+35501,4$

$+31470,4$

$+311825,4$

$+285742,1$

$+264129,1$

$+23569,0$

$+221142,9$

$+213733,6$

$+202923,9$

$+192541,1$

$+192540,4$

$+18482,9$

$+155452,6$

$+155453,7$

$+122751,8$
$+31614,0$
Lal. 28761.

Lal. 28834.

BZ. 421.

Mädler 2027.

BZ. 486.

BZ. 484 .

BZ. 484 .

BZ. 435 .

Lal. 38701.

BZ. 303.

BZ. 307, 315.

Lal. 40175.

BZ. 198.

BZ. 196.

BZ. 196.

Rümker 8757.

Lal. 40682.

Lal. 41258, 41259.

BZ. 22.

BZ, 28.
Comet III. 1862 . $\begin{array}{lllllll}a & 1 & 5 & 15,52 & -18 & 32 & 31,6 \\ b & 1 & 9 & 46,67 & -17 & 20 & 48,1\end{array}$

Bellona.

a $92224,49+135747,9$

b $9154,71+1580,4$

Victoria.

a $91448,47+22657,6$

Ariadne.

a $82311,44+144951,6$
Arg.-Oeltzen 680.

Arg.-Oeltzen 724.

BZ. 149.

BZ. 273.

BZ. 153 .

BZ. 62 . 
Sämmtliche Sternöpter sind hier obne die Reduction auf Wolfers' Tab. red. angegeher, bei den Positionen der Planeten und Cometen ist die Reduction jedoch angewandit. Wenn der Stern in mehreven Catalogen vorkünmt, ist immer das. Mittel aus ten verschiedenen Oertern angenommen.

Vergleichung der Beobachtungen mit strengen Ephemeriden.

43 Ariadne (Ephemeride im Jahrbuch).

$$
1861 \text { Oct. } 4 \underbrace{\Delta *}_{-690} \underbrace{-B \Delta \delta}_{-43^{1 / 2}}
$$

(14) Irene (Ephemeride im Jahrbuch)

Өct. $25+1,12-2,8$

$$
26+1,11-4,1
$$

(12) Victoria (Eptremeride in Jahroundh).

$$
\text { Nov. } 24+1,72+(24,0)
$$$$
25+2,02+6.7
$$

(3) Jưno (Ephemeride im Jahrbuch).

$$
\begin{aligned}
& \text { Nov. } 24-30,50-48,2 \\
& 25 \quad-30,42 \quad-52,1 \\
& \text { Dec. } 5 \quad-29,71 \quad-44,5 \\
& 6-29,64-46,9
\end{aligned}
$$

(40) Harmonia (Ephemeride im Jahrbuch).

$$
\begin{array}{rrr}
\text { Dec. } 12 & -28,48 & -53,9 \\
13 & -27,70 & -51,3 \\
14 & -27,28 & -55,0 \\
23 & -27,74 & -51,6
\end{array}
$$

Encke's Comet (1326, 1341).

$$
\begin{aligned}
& 1861 \text { Dec. } 23 \quad-12,80-21,4 \\
& 25-12,46-10,5 \\
& 1862 \text { Jan. } 10-24,24-I^{\prime} 59,8 \\
& 10-24,08-150,5 \\
& 22-44,91-548,2
\end{aligned}
$$

(9) Metis. (Epbemeride im Jahrbuch).

\begin{tabular}{|c|c|c|c|c|}
\hline 362 & Dec. 1 & + & 0, & $+5,2$ \\
\hline & 2 & 7 & 1,27 & $+\mathbf{3}, \mathbf{5}$ \\
\hline & 15 & $\vec{t}$ & 0,18 & $\begin{array}{r}+0,9 \\
-4,2\end{array}$ \\
\hline & 15 & 4 & 0,71 & $\begin{array}{r}5,0 \\
-\quad 5,\end{array}$ \\
\hline & 16 & - & 0,07 & $+6,6$ \\
\hline 1863 & Jan. 3 & + & $0 ., 08$ & $+8,5$ \\
\hline & 3 & - & 0,03 & $+4,9$ \\
\hline & 4 & + & 0,79 & 7,4 \\
\hline & 17 & + & 0,90 & $+7,2$ \\
\hline & 24 & + & 0,18 & 3,2 \\
\hline & 25 & 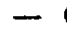 & 0,06 & 0,3 \\
\hline & 26 & - & 0,04 & 3,3 \\
\hline & 30 & - & 0,18 & 2,5 \\
\hline & Febr: 3 & + & 0,35 & 0,3 \\
\hline & 9 & 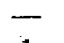 & 0,32 & 9,8 \\
\hline & 13 & + & 0,74 & 8,3 \\
\hline & 14 & & 0,57 & 5,4 \\
\hline & 17 & 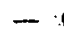 & 0,69 & 3,4 \\
\hline & 20 & & 0,20 & 7,0 \\
\hline & 21 & + & 0,02 & 15,0 \\
\hline & März 1 & & 0,71 & \\
\hline & 12 & & 0,56 & 27,9 \\
\hline
\end{tabular}

$$
\text { Feb. } 10-0,84+2,2
$$

(23) Thalia (Ephemeride im Jahrbuch).

$$
\begin{array}{rrr}
\text { Feb. } 19 & -7,82 & +47,1 \\
\text { März } 5 & -6,98 & +48,8 \\
8 & -7,21 & +43,4 \\
10 & -7,00 & +42,0
\end{array}
$$

(16) Psyche (Ephemeride im Jahrbuch).

$$
\begin{array}{rrr}
\text { Feb. 27 } & -16,94 & +81,3 \\
\text { März 8 } & -18,20 & +77,9 \\
10 & -17,66 & +78,2
\end{array}
$$

(29) Amphitrite (Ephenseride im Jahrbuch).

$$
\begin{aligned}
& \text { März } 8+0,99+6,0 \\
& 10+1,21+8,4 \\
& 12+1,14+3,3 \\
& 13+0,69+3,6
\end{aligned}
$$

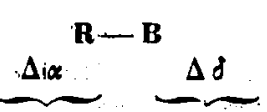

Fortunia (Ephemeride im Jahrhuch);

$$
\begin{array}{rrr}
\text { März } 8 & -11^{5.73} & +77^{4}: 8 \\
24 & -11,00 & +76,3 \\
29 & -10,77 & +78,8 \\
\text { April } 5 & -9,78 & +70,7
\end{array}
$$

(29) Euterpe (Ephemeride im Jahrhuch).

$$
\text { April } 5-2,16+7,4
$$

(39) Laetilia (Ephemeride im Jahrbuch).

$$
\text { Juni } 28+3,23+9,6
$$

Comet 1. 1863 (Ephemeride von Herrn Engelmann).

Bellona (Ephemeride im Jahrbuch).

Febr. $9-0,85-3,9$

$$
18-0,44-0,2
$$

Ariadne (Ephemeride im Jahrbuch).

Feb. $13-4,45-2,8$

Victoria (Ephemeride im Jahrbuch).

Febr. $9+2,21-2,6$

Bemerkungen zu den Beobachtungen.

Der:Comet II. 1861 war in October für den 6-f. Refractor schon recht schwach; ohwohl ich ilhn später noch oft sah, musste ich die Beobachtungen doch aufgeben.

Der Encke'sche Comet hatte am 23. Decbr. 1861 im dunkeln Felde $2 \frac{1}{2}-3^{\prime}$ Durchmesser, die grösște Verdichtung war etwas nördlich von der Mitle. December 25 hatte der Comet eine längliche Form, sein grösster Durchmesser war 3'. 1862 Jan. 10 hatte der Comet einen hellen Kern mit schwacher Nebelbülle und bei Mondschein nass diese im Durch- 
messer 13\%. Jan. 20 wan der Keen sehr. hell und präcise und machte auf das Auge den Eindruck eines Sternes 6-7 Grësse. Der Durchmesser der ganzen Nebelhülle betrug $1 \frac{1}{2}$. Der Venglaichstenn $b$ hat eine Eigenbewegung, welche ich nach dem. British Assqciation-Catabogue zu $0^{5} 021$ in $x$, $\neq 0^{\prime \prime} 0.5$ in d annahro.

Conket I1 1862 wwrde am 31. Juli van mir mit blossem Auge: gesebhen; ex hatte $1 \frac{1}{2}$ Durchmessen und eine schweifälunliehe Verlängerung uach der der: Sonne entgegengesetzten Bichlung, Ang. 1 erkannte ioh einen $1^{\circ}$ langen Schweif.

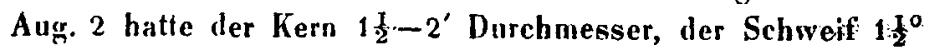
Länge. Ang. 5 war der Comet viel heller geworden. Aug. 27 zeigfe: sich ein selur deulicher Kern, von dem ein fächerförniger Sector ausging, der wieder mit einer Nebelmasse ungeben war. In der Nähe des Kerns war der Sector am hellsten. Aug, 28 war der Sectur viel undeutlicher, auch der Kern. katmk zu edkennen. Aug. 29 way der Kern wieder schärfer, auch der Sector gut zu erkennen. Der Schweif war im Cometensucher rechts viel hestimmter als links - er zeigte auf $\eta$ Urs. naj. Aug, 30 war wieder kein ordentlicher Kern zu erkennen, die redichtete Massc war sehr länglich und schmal, den Schweif verfolgte ich 12 Grad, seine Breite war am Enite über $1 \frac{1}{2}$ Grad.

Comet IH. 1862 erschien Dechr. 11862 ziemlich hell, so dass ich ihn in der Morgendämmerung noch gut sehen konnte, er glich einer verdichteten Nebelmasse von $1-1 \frac{1}{2}$ Durchmesser. Dec. 16 war er seines tiefen Standes wegen sehr schwach. Mitte Februar nach seinem Perihel suchte ich ihn bei sehr tiefem Stande in der Abenddämmerung mehrmals vergehens, an $18^{\text {ten }}$ und $20^{\text {sten }}$ sah ich ihn und beob. achtete ihn mit Vergleichstermen, welche ich aus Argelander's Zonen entnommen halke, von welchen der, eine aber nicht

Leipaig, im Mai 1863. der richtige zu sein scheint. Ich glauhes dass der Vergleichstern $b$ nicht der Argelander'sche Stern gewesen ist, den ich vorläufig angenonınen, sondern ein anderer Stern, dessen Position $15^{\mathrm{s}}$ in AR grösser und $4^{\prime}$ in. Deckination. südlicher ist. Die Beobachtungen vom 18. und 20. Febr siud wegen der Lichtsehwäche des Cometen vur mil grosser. Mühe erreicht.

Comet l. 1863 war bei seiner Entdeckung, 1862 Dec. 1, ein äusserst schwacher verwaschener Nebel von 2' Durchm;, Dec. 15 war er elwas beller gewonden; doch noch: sehr scbwex zu beobachten. 1863 Jan. 24. hatte der Comet cinen guten Kern und die Nehelhülle über 1' Durchm. Jan. 25 war der Kern nicht so gut begrenz.t als am Tage vorher, der Durchmesser desselben betrug mit 144facher Vergrösserung nur 11". Febr. 9 war der Kern sehr helt, die Nebelhülle war über 1' im Durchmesser, Febr. 13 war der scharf begrenate Kern auffallend hell und die Nebelhülle nach der von der Sonne abgewandien Seite grösser, als nach der entgegengesetzten; den läugsten Durchmesser der Nehelhụ̂He schätzte ich zu 1,'. Febr. 17 trat die elliptische Form der Nebelhïle noch mehr hervor, der Kern schien im Brennpunkte der Ellipse, die grosse Achse war 11,', die kleine 1'. März 1 war der Comet eine verwaschene Nebelmasse von $1 \frac{1}{2}$ 'Durchmesser, er hatte seh. an Helligkeit abgenommen. März 12 war er kaum noch zu erkennen und sehr schwer zu beobachten. Merkwïdig war die schnelle und hedeutende Zunahme des Lichtes nach dem Perihel. Mitte Februar hatte der Comet die grösste Helligkeit; eben so rasch, als sie zugenonmen, schien sie auch in März wieder abzunehnen. Die Vergleichung der Beobachtungen tieses Cometen mit der Ephemeride hat Herr Engelmann ausgefiihirt und selbiger wird auch nächstens die wahrscheinlichste Bahn, welche sich den bis jetzt bekanuten Beobachtungen anschliesst, ahgeleitet babew.

\section{Fortsetzung der Ephemeride der Diana (78). Von Herrn J. Spengler.}

Da Diana auf den. südlicher gelegenen Steruwarten noch etwas länger verfolgt werden kann, so erlauhe icly min, ejne Fort* setzung meiner Ephemeride für den Honat Juli zu geben:

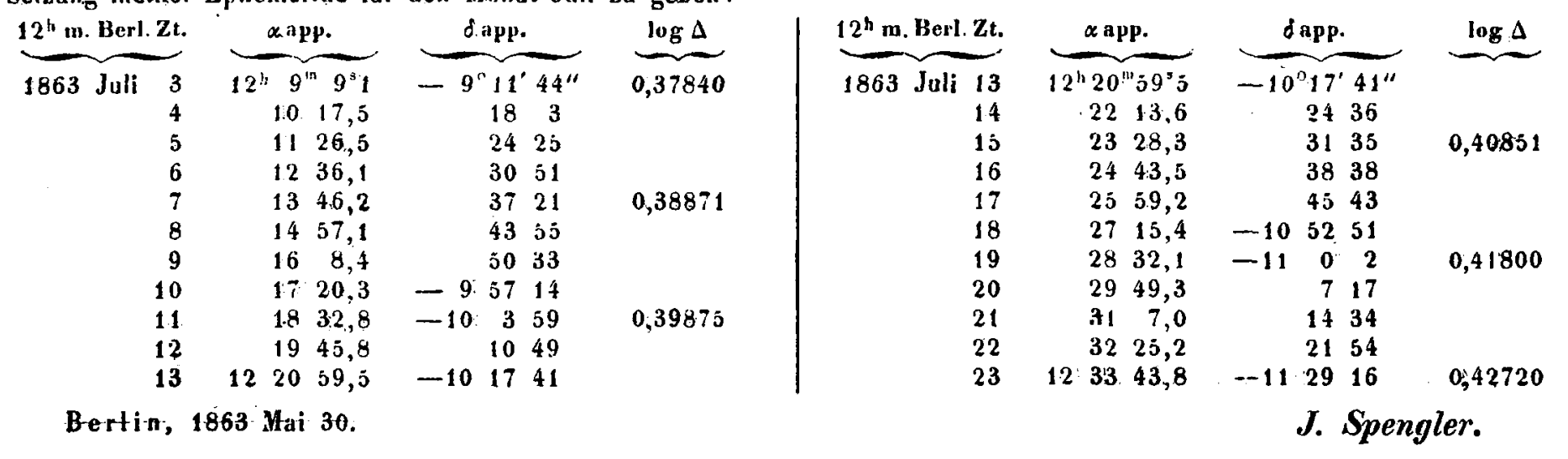

